\title{
Sauver le climat, mais pas chez soi ? Les conflits autour des installations de production d'énergie renouvelable en Allemagne
}

Saving the Climate-but not in my backyard - Disputes in Germany about sites designed for renewable energy production

Das Klima retten - aber nicht vor der eigenen Tür? Konflikte um Anlagen zur regenerativen Energieerzeugung in Deutschland

\section{Günter Weiss}

\section{OpenEdition}

\section{Journals}

Electronic version

URL: http://journals.openedition.org/rge/5467

DOI: $10.4000 /$ rge. 5467

ISSN: 2108-6478

\section{Publisher}

Association des géographes de l'Est

\section{Printed version}

Date of publication: 1 January 2015

ISSN: 0035-3213

\section{Electronic reference}

Günter Weiss, "Sauver le climat, mais pas chez soi ? Les conflits autour des installations de production d'énergie renouvelable en Allemagne », Revue Géographique de l'Est [Online], vol. 55 / n 1-2 I 2015, Online since 09 June 2015, connection on 08 September 2020. URL : http:// journals.openedition.org/rge/5467 ; DOI : https://doi.org/10.4000/rge.5467

This text was automatically generated on 8 September 2020

Tous droits réservés 


\title{
Sauver le climat, mais pas chez soi ? Les conflits autour des installations de production d'énergie renouvelable en Allemagne
}

\author{
Saving the Climate-but not in my backyard - Disputes in Germany about sites \\ designed for renewable energy production \\ Das Klima retten - aber nicht vor der eigenen Tür? Konflikte um Anlagen zur \\ regenerativen Energieerzeugung in Deutschland
}

Günter Weiss

\section{Introduction}

1 Le fait que les installations pour la production d'énergies renouvelables soient souvent une source de conflits (cf. photo 2), est maintenant avéré (par exemple Hollersen 2010) et est l'objet de recherches scientifiques (voir Bosch et Peyke 2011). La politique de développement forcé des énergies, renouvelables, principalement motivée par la protection du climat, est fortement entravée dans son action par une résistance surgissant de toutes parts ; d'autant plus que presque tous les types de centrales, ainsi que les nouvelles lignes de transport d'électricité se retrouvent au centre de la critique.

On qualifie souvent cette attitude critique de NIMBY (« pas dans mon jardin») ; ce qui signifie que les citoyens n'ont rien contre une technologie particulière en elle-même, mais ne veulent pas la voir installée devant chez eux (Jones et Reiser 2010). Pour résoudre ces problèmes, la stratégie consiste à accélérer le processus d'approbation, en réduisant la participation des citoyens, ainsi qu'en cherchant à impliquer un plus grand nombre de personnes dans les processus d'approbation, par exemple à travers une participation aux gains provenant des énergies renouvelables (cf. Rau / Walter / Zoellner, 2011). 
$3 \quad$ Alors qu'il existe des représentations claires des potentialités des espaces naturels pour tel ou tel type de ressource énergétique, la confusion règne en ce qui concerne l'importance des contextes régionaux pour leur acceptation ; en l'occurrence, la question de savoir s'il y a des endroits particulièrement exposés à des conflits pour la production d'énergie renouvelable. L'analyse des conflits autour des installations industrielles polluantes a déjà pu montrer que le développement des conflits d'usage, en principe est aléatoire et donc qu'il n'y a pas a priori de zone sans problème ou au contraire sujette aux conflits (Weiss 2008, p 429).

4 La référence à l'espace des conflits autour des implantations pour la production d'énergie renouvelable est l'objet de l'analyse suivante ; à savoir la question de l'importance de l'emplacement de l'installation dans l'argumentation des acteurs impliqués dans les conflits d'usage. Il ne s'agit donc pas des qualités «objectives» de localisation, statistiquement évaluables (cf. Conseil allemand pour la gestion du paysage 2006), mais des problèmes perçus par la population tels qu'ils s'articulent concrètement dans les litiges.

\section{L'identification des conflits d'usage de l'espace}

5 L'identification des conflits d'usage n'est pas sans poser problème. Même si on se limite aux conflits manifestes, de notoriété publique, il est nécessaire d'un point de vue méthodologique que les déclarations ne soient pas seulement orales et donc éphémères, mais aussi documentées afin de pouvoir être examinées avec le recul nécessaire. Un deuxième critère est que le conflit doit avoir atteint une certaine «masse critique», afin de ne pas surestimer les déclarations simples telles que les courriers ou les forums en ligne. En fonction de ces deux critères, les conflits pris en considération dans cet article doivent être portés par au moins un groupe organisé, généralement une initiative citoyenne. En général, seule l'existence d'une telle organisation permet de faire ressortir des arguments et des opinions ayant une certaine persistance. Les initiatives citoyennes trouvent un écho dans la presse locale et régionale et disposent assez souvent de sites internet qui leur servent de tribune pour exprimer leurs préoccupations. La plupart rassemblent aussi toutes sortes de documents sur le conflit (récit des événements, articles de journaux, rapports, dépliants, etc.). On exclue ici les conflits intra- et intercommunaux, qui se déroulent principalement au niveau des conseils municipaux, car ces conflits relèvent de la routine de la démocratie parlementaire et ne reflètent pas nécessairement les opinions de la population locale. 
Figure 1 : parcs photovoltaïques (points jaunes) et centrales de pompage-turbinage (points bleus) contestés en Allemagne (2009-2012)

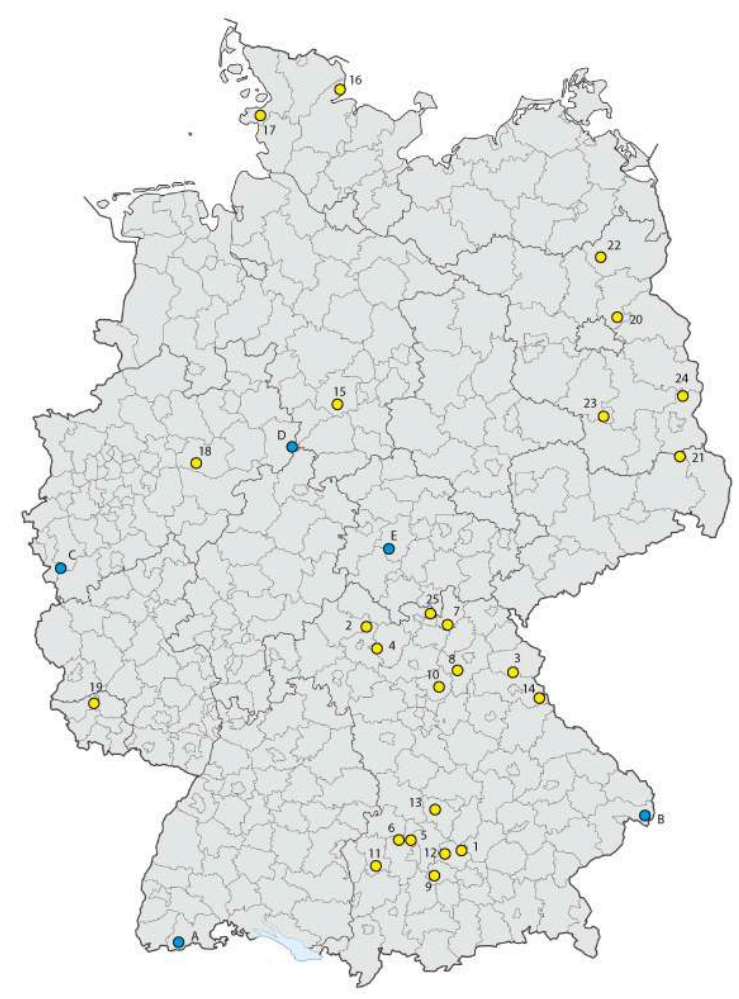

Source: G. Weiss

6 L'analyse qui suit portera sur les parcs photovoltaïques au sol et les centrales hydroélectriques de pompage-turbinage à réservoir (fig. 1). Les deux systèmes sont représentatifs d'un type particulier d'installation en termes de technologie, d'importance économique et de taille: d'une part plutôt décentralisée, la production d'énergie à petite échelle (tout comme les éoliennes, les centrales à biomasse); d'autre part plus centralisée, la production d'électricité et le stockage à grande échelle. Alors que les installations photovoltaïques au sol servent à la production immédiate d'électricité à partir du soleil et se distribuent de manière décentralisée dans les régions à insolation relativement élevée (Brücher 2009, p 200), les centrales de pompage-turbinage correspondent à un stockage temporaire d'énergie pour l'électricité et nécessitent un relief de grande énergie. Dans les centrales de pompageturbinage on utilise les surplus d'électricité bon marché pour pomper l'eau d'un niveau inférieur à un bassin supérieur. En cas de besoin, on fait de nouveau s'écouler l'eau du réservoir supérieur vers le bassin inférieur à travers des turbines, afin de produire du courant de pointe coûteux (voir Trianel 2011). 
Figure 2 : évolution de la capacité installée en énergies renouvelables en Allemagne

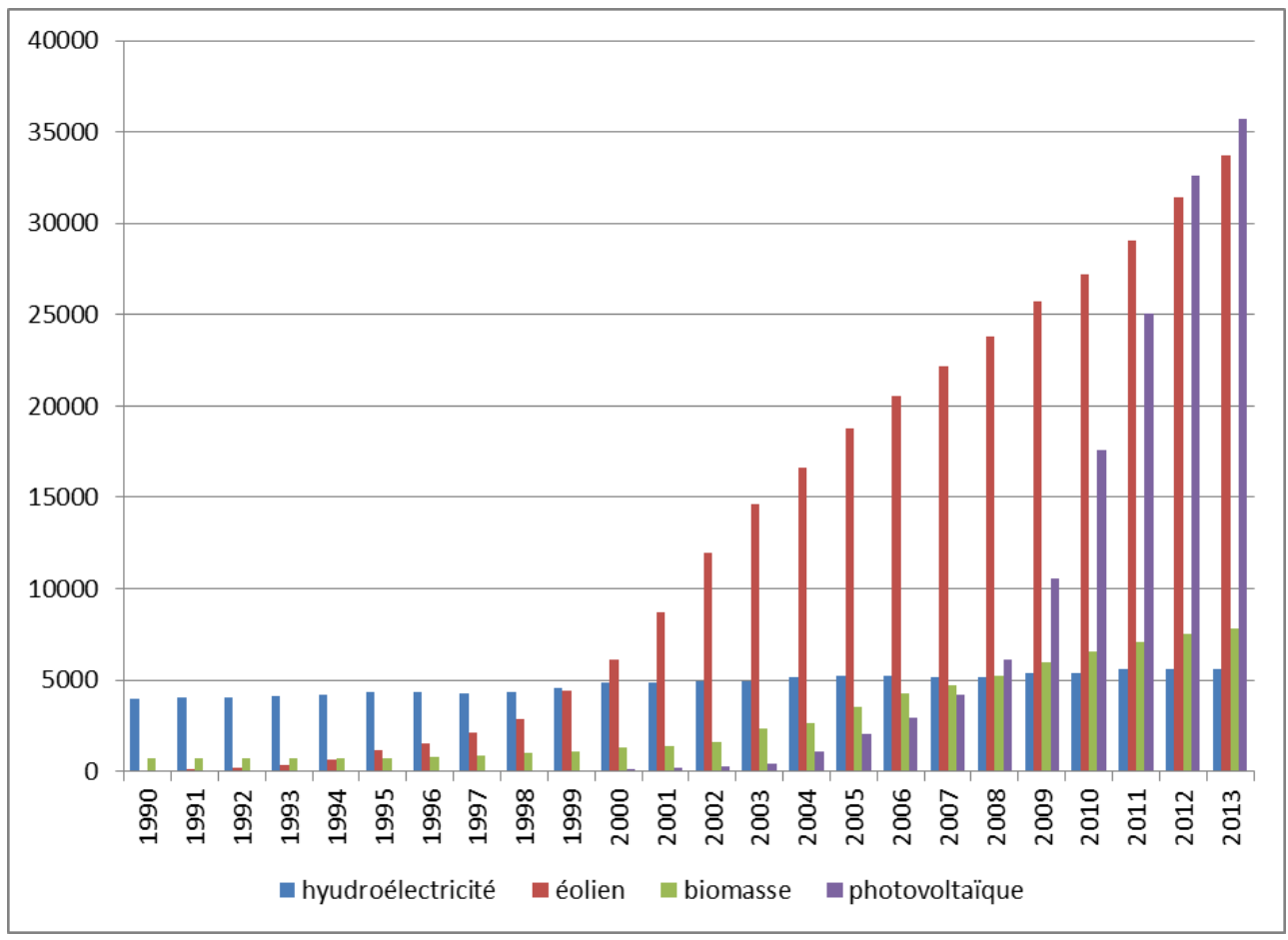

Tableau 1 : évolution de la capacité installée en énergies renouvelables en Allemagne depuis 1990

\begin{tabular}{|l|l|l|l|l|l|l|l|}
\hline & hydro & éolien & Biomasse & $\begin{array}{l}\text { partie biologique } \\
\text { des ordures }\end{array}$ & Qhoto-valtaïque & géo-thercoie & $\begin{array}{l}\text { capacité } \\
\text { totale }\end{array}$ \\
\hline 1990 & 3429 & 55 & 85 & 499 & 1 & 0 & 4069 \\
\hline 1991 & 3394 & 106 & 96 & 499 & 2 & 0 & 4097 \\
\hline 1992 & 3550 & 174 & 105 & 499 & 3 & 0 & 4331 \\
\hline 1993 & 3509 & 326 & 144 & 499 & 5 & 0 & 4483 \\
\hline 1994 & 3563 & 618 & 178 & 499 & 6 & 0 & 4865 \\
\hline 1995 & 3595 & 1121 & 215 & 525 & 8 & 0 & 5464 \\
\hline 1996 & 3510 & 1549 & 253 & 551 & 11 & 0 & 5874 \\
\hline 1997 & 3525 & 2089 & 318 & 527 & 18 & 0 & 6476 \\
\hline 1998 & 3601 & 2877 & 432 & 540 & 23 & 0 & 7473 \\
\hline 1999 & 3523 & 4435 & 467 & 555 & 32 & 0 & 9012 \\
\hline 2000 & 3538 & 6097 & 579 & 585 & 76 & 0 & 10875 \\
\hline 2001 & 3538 & 8750 & 696 & 585 & 186 & 0 & 13755 \\
\hline 2002 & 3785 & 11989 & 843 & 585 & 296 & 0 & 17498 \\
\hline 2003 & 3934 & 14604 & 1091 & 847 & 435 & 0 & 20911 \\
\hline 2004 & 3819 & 16623 & 1444 & 1016 & 1105 & 0,2 & 24007 \\
\hline 2005 & 4115 & 18390 & 1964 & 1210 & 2056 & 0,2 & 27735 \\
\hline 2006 & 4083 & 20579 & 2620 & 1250 & 2899 & 0,2 & 31431 \\
\hline 2007 & 4169 & 22194 & 3434 & 1330 & 4170 & 3,2 & 35300 \\
\hline 2008 & 4138 & 23836 & 3969 & 1440 & 6120 & 3,2 & 39507 \\
\hline 2009 & 4151 & 25716 & 4519 & 1550 & 9914 & 7,5 & 45858 \\
\hline 2010 & 4395 & 27209 & 5014 & 1650 & 17320 & 7,5 & 55596 \\
\hline 2011 & 4469 & 28730 & 5744 & 1486 & 25039 & 7,5 & 65806 \\
\hline 2012 & 4448 & 30869 & 6157 & 1400 & 32643 & 12,1 & 75965 \\
\hline
\end{tabular}

Source : Erneuerbare Energie in Zahlen

7 Le développement de la capacité installée des éoliennes en Allemagne (voir Figure 2 et tableau 1) fait que l'hydroélectricité, en stagnation depuis 2003, connaît de nouveau 
une légère augmentation. Depuis 2003, la capacité photovoltaïque augmente continuellement, en raison du tarif d'achat garanti par la loi EEG sur les énergies renouvelables et de la baisse des prix des modules solaires. L'abaissement annoncé du tarif d'achat à partir de 2009 a conduit en 2008 à un boom des installations photovoltaïques, tandis que la nouvelle réduction pour 2012 a généré également un boom en 2011.

Parmi les différents critères possibles d'une analyse des conflits on doit ici retenir les arguments des parties prenantes du conflit et plus particulièrement la référence à l'espace. En fait, les critères pertinents tels que les motivations et les constellations d'acteurs, les modes d'acquisition des connaissances et la planification stratégique restent souvent dissimulés. Les arguments exposés ici se rapportent à une analyse qualitative de documents disponibles sur Internet, provenant de la presse quotidienne et des initiatives citoyennes locales ou régionales. Ont été examinés 6 projets de centrales de pompage-turbinage et 28 installations photovoltaïques au sol pour la période allant de 2009 à 2012.

\section{Typologie des arguments et des conflits}

Les arguments utilisés dans les conflits observés peuvent être divisés en quatre groupes

1 ) les avantages ou inconvénients de l'installation ou de la technologie utilisée ainsi que les conséquences directes liées à l'exploitation des installations (par exemple la construction de lignes électriques de connexion au réseau). Cela comprend aussi les impacts temporaires associés à la construction des installations.

11 2) les avantages et les inconvénients de l'installation en relation avec la voie de développement telle qu'elle est perçue régionalement ou localement. Ici, il s'agit de voir si le système s'adapte ou non au cadre régional. Ce cadre peut être de nature différente, par exemple suivant les priorités économiques régionales et les traditions, mais aussi les charges cumulées. En outre, il faut aussi compter sur les «modèles" régionaux, à savoir des projets similaires qui peuvent être responsables de la réussite ou de l'échec d'un système ou d'une stratégie.

12 3) les avantages et les inconvénients qui sont associés à certaines alternatives régionales de localisation, dans la mesure où, par rapport à un site d'implantation privilégié, il existe des options alternatives. Cela comprend les arguments en ce qui concerne la taille de l'implantation, la combinaison avec d'autres équipements et les mesures compensatoires nécessaires. Les arguments de ce type sont souvent considérés dans la discussion comme typiques de l'attitude NIMBY.

13 4) Les avantages et les inconvénients du système par rapport aux points forts du débat sur la politique énergétique. Les débats et les prévisions sur les possibles évolutions alternatives peuvent jouer un rôle important. Une nouvelle installation peut ainsi jouer en faveur du développement, mais aussi se révéler être un mauvais investissement, si le développement économique et technologique suit finalement une autre voie.

Il est en outre possible de distinguer deux types de conflits :

15 1) les "conflits locaux». Dans ce cas, l'installation de production énergétique est principalement mise en rapport avec le cadre de vie de la population locale : le système est considéré comme étant une perturbation de la qualité du cadre de vie et de loisir. 
Dans le contexte d'une violation du droit à un environnement intact, les autres contextes régionaux comme par exemple les voies locales de développement économique ne comptent pas et sont parfois utilisés comme arguments secondaires. Les constellations d'acteurs comprennent généralement d'une part, un groupe de citoyens critiques (une initiative citoyenne) et de l'autre les investisseurs et le conseil municipal. 2) les «conflits régionaux». Dans ce type de conflit, les nuisances pour l'environnement résidentiel peuvent certes être prises en compte, mais la plupart des installations correspondantes sont en fait situées loin des zones résidentielles. Au contraire, en raison de leur taille elles sont perçues comme étant d'importance régionale et par conséquent considérées comme des formes préférentielles de développement local. Leur importance se reflète également dans la participation d'acteurs politiques et administratifs dépassant le cadre local (Kreis, gouvernement du Land) et d'ONG (par exemple la Fédération de protection de la nature BUND, l'organisation de protection des forêts allemandes, le club Rennsteig).

Photo $1 \mathrm{a}$ : le bassin supérieur de la centrale de pompage-turbinage de Goldisthal

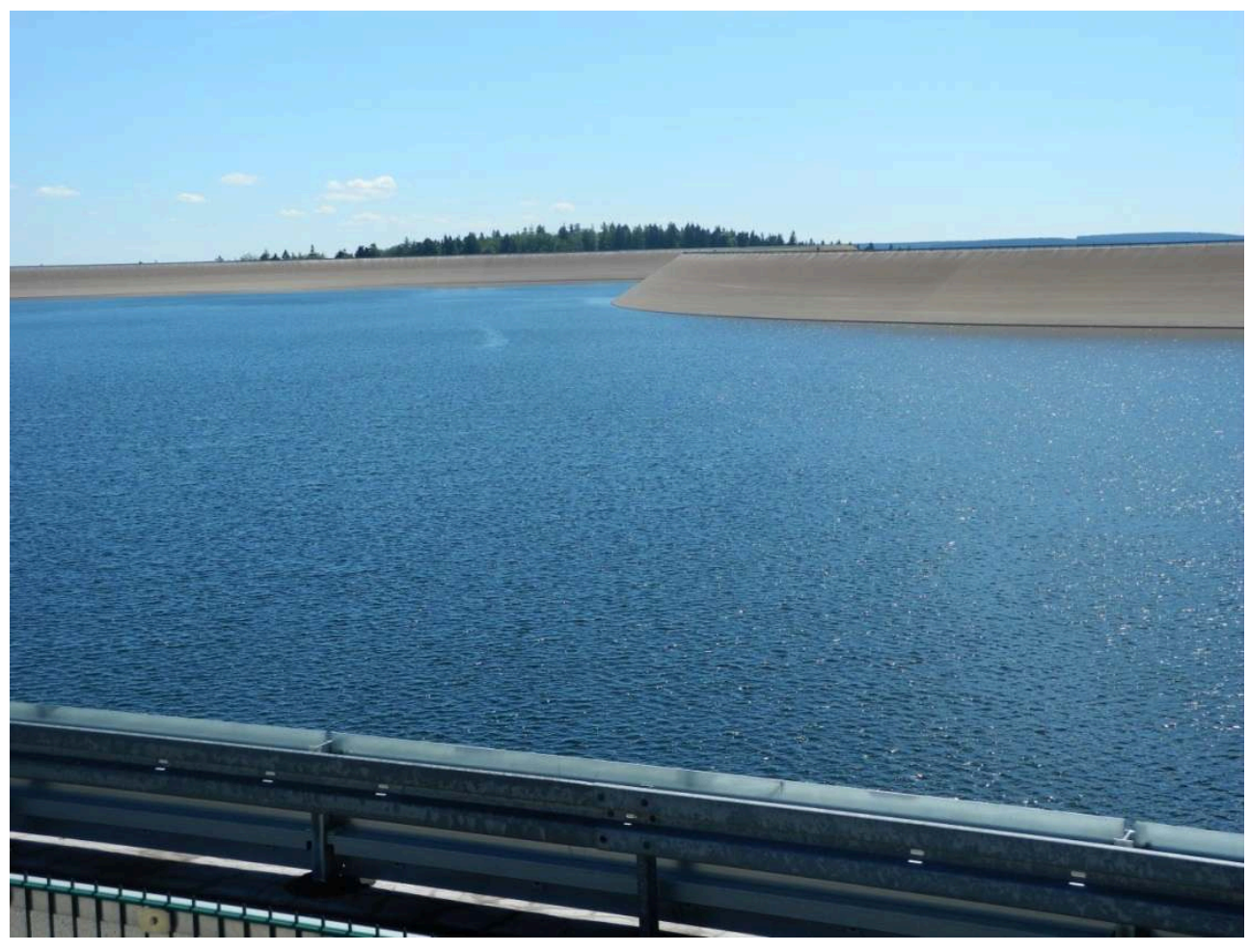


Photo $1 \mathrm{~b}$ : bâtiment de l'administration municipale de Goldisthal

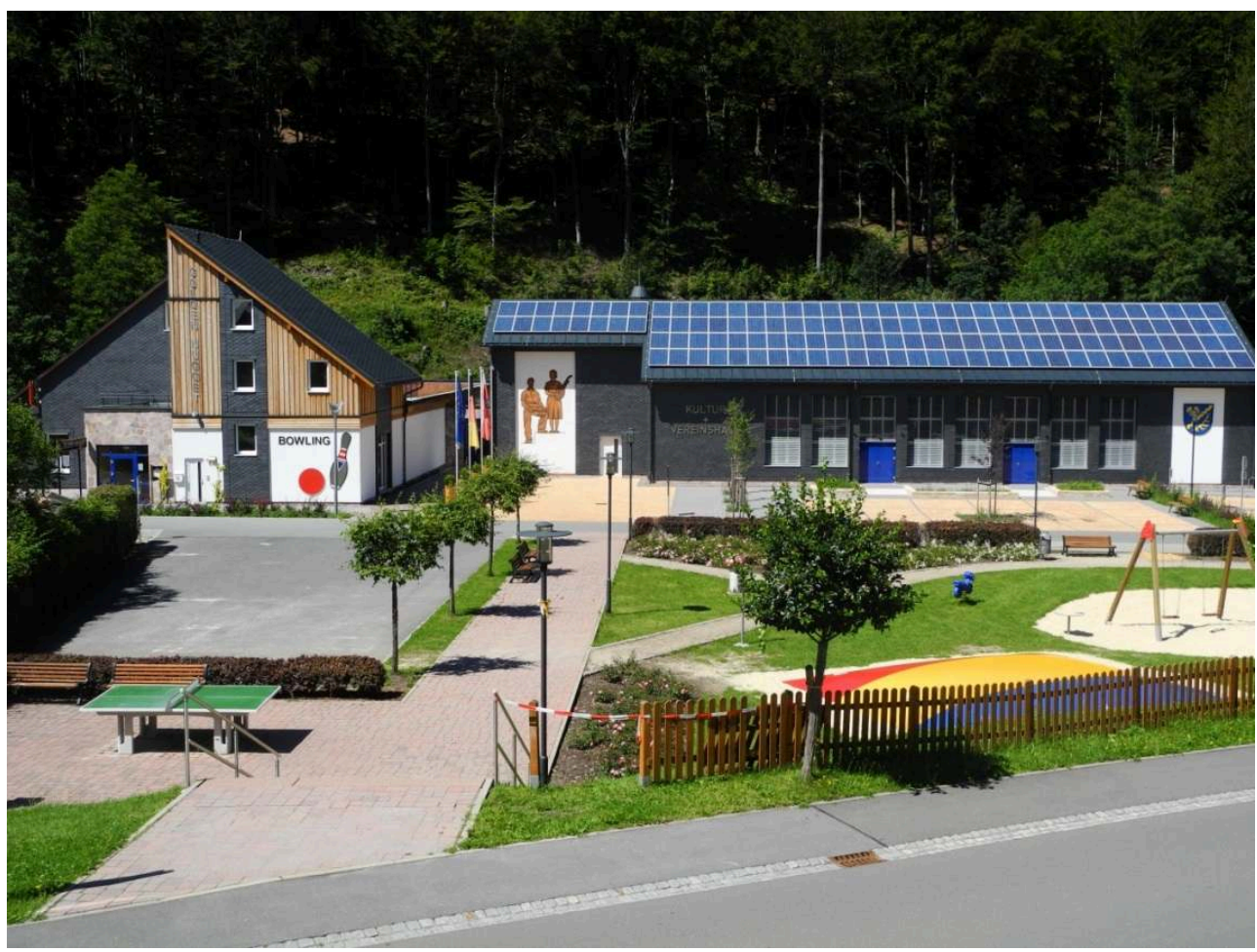

Photos 1a et $1 \mathrm{~b}$ : L'enrichissement par les énergies renouvelables ? Le bassin supérieur de la centrale de pompage-turbinage de Goldisthal en Thuringe et la rénovation du bâtiment de l'administration municipale de Goldisthal qui a été possible grâce aux revenus des taxes de la centrale hydroélectriques.

\section{L'exemple des centrales de pompage-turbinage}

17 Les différends autour des centrales de pompage-turbinage sont caractéristiques des "conflits régionaux» (voir figure 3). Les avantages spécifiques qui sont mis en avant dans les débats concernent l'absence d'émissions et la faible atteinte aux paysages car les installations de la centrale sont souterraines, si bien que seuls les deux bassins sont visibles de l'extérieur. La technologie est considérée comme mature et fiable; les profits générés profitent à la commune locale sous forme de recettes fiscales. Les inconvénients du système sont principalement liés à l'utilisation des terres pour la construction des deux bassins. Le réservoir supérieur qui est soumis à de fortes fluctuations quotidiennes du niveau de l'eau, ne peut pas être utilisé à d'autres fins et doit être clôturé (photo 1a); dans le bassin inférieur les fluctuations du niveau de l'eau peuvent également affecter l'utilisation et l'écologie aquatique près de la rive. Avec les nouvelles surfaces aquatiques, il faut aussi compter avec des changements dans le microclimat et l'hydrographie locale. La réalisation des lacs et des parties souterraines de l'usine implique une longue période de construction avec d'importants déplacements de matériel, de sorte qu'il existe des nuisances causées par une exposition au bruit, à la poussière et aux vibrations. Le stockage de l'excès de déblais et la réalisation d'une nouvelle ligne électrique de connexion au réseau constituent d'autres formes d'occupation des terres découlant de ces travaux. 

dans la forme de développement local est souvent controversée (photos $2 a$ et $2 b$ ). Ainsi, le système peut être considéré comme incompatible avec une voie de développement axée sur le tourisme, car il fait fuir les touristes en particulier par les nuisances dans la période de construction, mais aussi plus tard par les fluctuations des niveaux d'eau et les zones de restriction qui en résultent. Tout aussi critique est la perception de la région comme un «site d'infrastructures encombrantes», telles que les centrales existantes, les autoroutes, etc. Ici, il convient de faire en sorte que la nouvelle installation ne renforce pas la voie considérée comme problématique. En revanche, la centrale de pompage-turbinage est considérée comme conforme à la situation d'une " région à problèmes économiques " parce que des emplois sont créés par la construction et l'exploitation et plus tard par des recettes fiscales non négligeables (photo 1b). En ce qui concerne la voie de développement comme "paysage énergétique », la centrale de pompage-turbinage est également considérée comme appropriée, puisque les points forts existants connaissent une nouvelle expansion. Les usines de pompage-turbinage existantes, ainsi que les centrales hydroélectriques au fil de l'eau et les systèmes photovoltaïques sont des éléments du paysage de l'énergie dont les surplus pourraient être stockés dans la centrale.

19 L'intégration du projet dans le débat sur la politique énergétique se révèle être d'une importance essentielle dans la discussion sur les centrales de pompage-turbinage. Ses promoteurs soulignent l'importance du pompage comme technologie mature et éprouvée pour stocker l'électricité lors de la demande de pointe ; ce qui est absolument nécessaire pour compenser la production de sources d'énergie intermittentes comme le vent et l'énergie photovoltaïque. En tant que tel, elles sont considérées comme un élément indispensable de la transition énergétique et comme une composante du nouveau système d'énergie renouvelable. La critique s'exprime sur un changement de perspective concernant l'avenir de l'économie de l'énergie. Les opposants considèrent les installations de pompage comme une technologie dépassée et sont en faveur d'une production décentralisée et d'un stockage de l'énergie, que ce soit par la cogénération, le chargement et le déchargement de batteries de véhicules électriques, le stockage de l'électricité sous forme d'hydrogène, dans le réseau de gaz naturel ou dans des réservoirs souterrains d'air comprimé, par les réseaux intelligents ou encore la baisse de la consommation d'énergie. D'autres critiques soulignent qu'en raison de l'utilisation croissante du photovoltaïque, la demande de courant en période de pointe, dans les heures de midi, est déjà en baisse. D'après ces considérations, les centrales de pompage-turbinage seront, au mieux, une technologie de transition ; ce qui compte tenu de leur longue période de construction, fait courir le risque qu'elles soient déjà superflues ou non rentables lors de leur mise en service, parce que la rentabilité de ces systèmes est basée sur une assez grande différence de prix entre les périodes d'excédent et de pic de consommation d'électricité. Comme en raison de l'ampleur des investissements nécessaires ces installations ne peuvent être réalisées que par des grandes entreprises ou des consortiums, elles sont critiquées comme étant des «dinosaures» de l'ancienne époque de l'énergie centralisée non durable. 
Photo 2a : affiche « sauvez le Rennsteig »

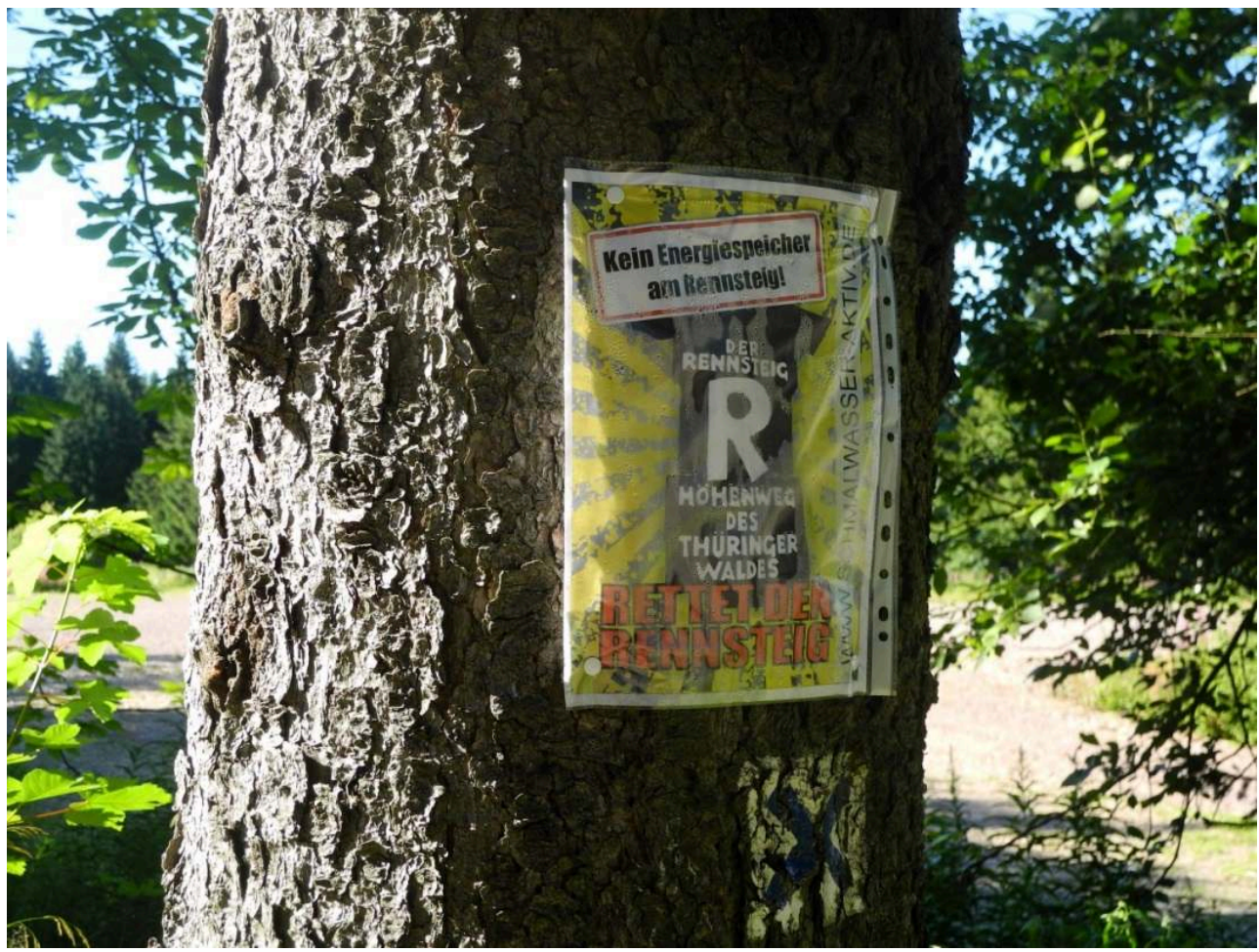

Photo $2 \mathrm{~b}$ : affiche « parc énergétique ou parc naturel ? »

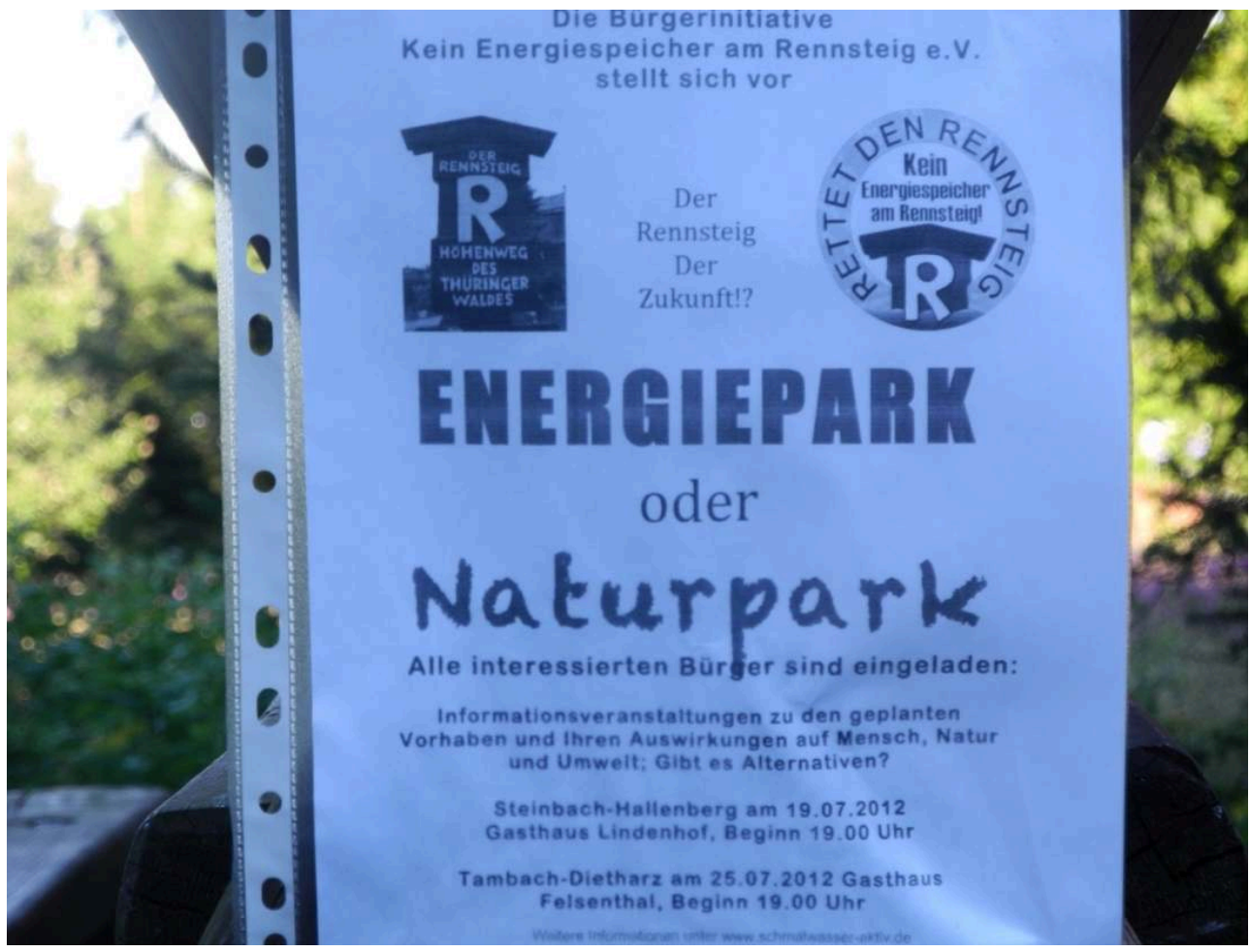

Problèmes avec les énergies renouvelables ? Deux affiches contre la centrale de pompage-turbinage de Schmalwasser en Thuringe avec le bassin supérieur à proximité du Rennsteig, le célèbre sentier de randonnée de la Forêt de Thuringe. 
Tableau 2 : projets de parcs photovoltaïques contestés (2009-2012)

\begin{tabular}{|c|c|c|c|c|c|}
\hline $\mathrm{Nr}$. & semmune & Kreis & Bundesland & $\begin{array}{l}\text { capacité } \\
{[\mathrm{MW}]}\end{array}$ & $\begin{array}{l}\text { surface } \\
\text { [ha] }\end{array}$ \\
\hline 1 & Petershausen & Dachau & Bayern & 10 & 32 \\
\hline 2 & Thunderf & Bad Kissingen & Bayern & - & 44 \\
\hline 3 & Windischeschenbach & Neustadt/Waldnaab & Bayern & - & 8 \\
\hline 4 & Theres & Hassberge & Bayern & 19 & $2 \times 25$ \\
\hline 5 & Gablingen & Augsburg & Bayern & - & 12,6 \\
\hline 6 & Heretsried & Augsburg & Bayern & - & 9 \\
\hline 7 & Weißenbrunn & Kronach & Bayern & - & 5,3 \\
\hline 8 & Aherntal & Bayreuth & Bayern & - & 2,2 \\
\hline 9 & Kottgeisering & Fürstenfeldbruck & Bayern & - & 3 \\
\hline 10 & Pinzberg & Forchheim & Bayern & 4,07 & 12,5 \\
\hline 11 & Krumbach & Günzburg & Bayern & - & - \\
\hline 12 & Markt Indersdorf & Dachau & Bayern & - & 2,5 \\
\hline 13 & Bohrenfeld & Neuburg-Schrobenhs. & Bayern & - & 9 \\
\hline 14 & Eslarn & Neustadt/Waldnaab & Bayern & 3,2 & - \\
\hline 15 & Sibbese & Hildesheim & Niedersach & 1,3 & 4 \\
\hline 16 & Brodersby & Rendsburg-Eckernför & Schlesw.-H & - & 3,5 \\
\hline 17 & Katharinenheerd & Husum & Schlesw.-H & 3,5 & 10,8 \\
\hline 18 & Möhnesee & Soest & NRW & - & 3,5 \\
\hline 19 & Weiskirchen & Merzig-Wadern & Saarland & 30 & ca. 70 \\
\hline 20 & Werneuchen & Barnim & Brandenbg & - & 178 \\
\hline 21 & Spreetal & & Sachsen & - & - \\
\hline 22 & Templin & Uckermark & Brandenbg & 128 & 214 \\
\hline 23 & Dahme & Teltow Fläming & Brandenbg & 5 & 15 \\
\hline 24 & Schenkendöbern & Spree-Neiße & Brandenbg & 250 & 650 \\
\hline 25 & Neustadt b. Coburg & Coburg & Bayern & 3,4 & 14,3 \\
\hline
\end{tabular}

Tableau 3 : projets contestés de centrales de pompage-turbinage (2009-2012)

\begin{tabular}{|l|l|l|l|l|l|l|}
\hline Nr. & commune & Kreis & Bundesland & $\begin{array}{l}\text { capacité } \\
{[\mathrm{MW}]}\end{array}$ & $\begin{array}{l}\text { surface du } \\
\text { bassin } \\
\text { supérieur } \\
\text { [ha] }\end{array}$ & $\begin{array}{l}\text { surface } \\
\text { du bassin } \\
\text { inférieur } \\
\text { [ha] }\end{array}$ \\
\hline A & Bad Säckingen & Waldshut & Baden-Wü. & 1.400 & 14 & - \\
\hline B & Untergriesbach & Passau & Bayern & 300 & 24 & - \\
\hline C & Simmerath & Aachen & NRW & 640 & 50 & - \\
\hline E & Tambacb-Dietharz & Gotha & Thüringen & 1.070 & - & - \\
\hline
\end{tabular}

\section{L'exemple des systèmes photovoltaïques au sol}

Les débats sur les fermes solaires présentent généralement les caractéristiques des " conflits locaux » (voir figure 4). Les défenseurs de ces installations mettent en avant les avantages tels que l'absence d'émissions (bruit, odeur, dioxyde de carbone) et la faible consommation réelle de terrain, étant donné que seule une petite partie de la surface d'un parc solaire est effectivement construite. Sous les panneaux solaires il est généralement possible de pratiquer une agriculture extensive; ce qui comparé à l'agriculture intensive représente une revalorisation écologique : la fertilisation et les traitements par pesticides disparaissent, la diversité des espèces d'insectes et d'oiseaux augmente. De plus, grâce à la mise en place d'une ceinture verte, les installations sont à peine visibles de l'extérieur. Ces installations contribuent aussi à la diversification et donc à l'amélioration des revenus des agriculteurs qui, en raison de l'écart grandissant entre les coûts de production et les revenus, sont confrontés à l'avenir incertain de la production agricole traditionnelle. La municipalité locale bénéficie aussi de recettes fiscales provenant des revenus des propriétaires fonciers et des exploitants. 
21 On critique par contre les inconvénients concernant la dégradation du paysage, surtout si l'installation est située en un lieu à forte visibilité, ou en bordure des lieux habités. Cela peut aller d'une dépréciation de la valeur résidentielle et récréative à une dévaluation mobilière et immobilière. On mentionne souvent une perte de précieuses terres agricoles et une stérilisation des surfaces, puisque la zone de l'installation est clôturée et n'est plus accessible aux hommes comme aux animaux les plus gros. Avec l'utilisation dans les modules de matériaux au tellurure de cadmium, il y a un risque de contamination des sols par du cadmium hautement toxique lors de dégâts dus aux tempêtes, d'incendies, ou de la démolition des panneaux. On suspecte également des dangers pour la santé à cause du rayonnement (éblouissement), de la pollution électromagnétique et du bruit du système de l'onduleur. En fin de compte, on discute aussi des problèmes économiques : Le rendement est tributaire des conditions météorologiques, de la pollution de l'air et des réparations ; les revenus de la taxe professionnelle dépendent du siège de la société d'exploitation. Les modules tout comme la construction du parc photovoltaïque sont rarement réalisés par des entreprises locales, mais plutôt par des consortiums spécialisés de dimension suprarégionale.

Être intégré dans un contexte de voies de développement régional est presque hors de propos pour les critiques. On déplore rarement une altération de la fonction touristique et de loisirs. Le projet est plutôt lié à un contexte local, à savoir la fonction résidentielle et de loisirs d'une zone, y compris la valeur immobilière. On préfère donc interpréter le parc solaire comme étant une " installation industrielle " ou une " centrale " incompatible avec des zones résidentielles rurales.

23 La référence explicite à la discussion sur la politique énergétique apparaît en outre comme un argument secondaire. Pour les investisseurs et les propriétaires, mais aussi du point de vue de la politique municipale, les revenus et les impôts provenant des installations viennent avant la contribution du photovoltaïque à l'approvisionnement énergétique durable au regard de l'épuisement des combustibles fossiles et des problèmes environnementaux (changement climatique). Les critiques considèrent que deux points forts du débat sur la politique énergétique sont pertinents : d'une part, le débat sur les subventions solaires, qui donne l'impression que l'énergie solaire est excessivement subventionnée, ou du moins que son existence dépend fortement des subventions. Cela vient conforter l'hypothèse selon laquelle quelques bénéficiaires (les développeurs, les opérateurs, les propriétaires fonciers) bénéficient de l'argent des taxes et des coûts excessifs imposés aux clients privés; de telle sorte que selon les mots d'un critique, on assiste à «une redistribution du bas vers le haut», ou que le paysage est sacrifié au "profit". Deuxièmement, on retrouve dans l'argumentation locale les débats politiques sur les voies alternatives de développement du photovoltaïque : la restriction des implantations aux toitures, aux friches industrielles et aux paysages en voie de réhabilitation (décharges, mines), ou alors déplacer la production électrique solaire dans les zones ayant une beaucoup plus forte insolation. Il s'agit en l'occurrence du projet «Desertec» en Afrique du Nord.

24 Seul le conflit sur le parc solaire "Lieberose Heide " en Brandebourg présente les caractéristiques d'un conflit régional : avec une superficie d'environ 650 ha et une puissance de $250 \mathrm{MW}$, il s'agit d'un grand projet situé sur une zone de conversion située au milieu des bois. Il est considéré plutôt négativement par les acteurs, principalement au regard d'une voie régionale de développement touristique axée sur une "exposition 
internationale des forêts" par opposition à l'époque précédente marquée par le paysage énergétique de l'exploitation du lignite.

\section{Les conflits d'usage résultant des discussions sur la politique énergétique}

En résumé, tous les conflits observés montrent l'importance plus ou moins centrale du débat sur la politique énergétique. Avant la «transition énergétique» de 2011, favoriser les énergies renouvelables pour l'avenir était en effet largement consensuel ; mais le débat politique portait sur la question des subventions, en particulier sur le niveau et les conditions du tarif d'achat. Cela a donné l'impression reflétée par l'argumentation employée dans les conflits locaux, que l'on a subventionné au détriment des contribuables et des consommateurs d'électricité, des installations de production d'énergie techniquement complexes, ou non rentables. On peut se référer ici aux subventions pour le solaire, qui en raison de l'expansion des systèmes photovoltaïques a à son tour conduit à une réduction de la politique de subvention: les systèmes photovoltaïques sur surfaces agricoles n'ont plus bénéficié du tarif d'achat et les subventions en faveur du solaire ont été successivement réduites. Cela veut dire que la technologie initialement favorisée n'était pas si bénéfique qu'il y paraissait. Les paroles des citoyens reflètent manifestement une certaine perplexité : " Sur la route du 21ème siècle il y a de nombreuses impasses " dit un citoyen. Faire des parcs solaires sur de bonnes terres agricoles face à la pénurie alimentaire mondiale, l'application de pesticides et d'engrais pour les cultures énergétiques de maïs, ou l'arrêt forcé des éoliennes en périodes de surproduction de courant donnent l'impression d'une évolution déraisonnable, contraire au bon sens. L'incertitude de la position de la population locale en ce qui concerne les parcs solaires et les centrales de pompageturbinage peut être aussi illustrée par deux autres aspects : le caractère aléatoire du conflit et l'incertitude de son issue. En fin de compte, il suffit souvent d'un petit nombre de citoyens mécontents pour déclencher un conflit. Bien que l'on puisse confirmer l'observation parfois exprimée par les intervenants selon laquelle, les installations éloignées des lieux habités sont moins contestées, tous les systèmes situés à proximité des habitations ne sont pas pour autant à l'origine de conflits. Cependant, les votes dans les conseils municipaux et les pétitions de citoyens montrent souvent des résultats proches pour ou contre un projet. Les conseils municipaux ont tendance à d'autant plus favoriser une installation qu'elle est grande et donc que les recettes fiscales peuvent être importantes.

Dans les conflits qui se sont développés après l'accident de Fukushima et à la suite de la décision du gouvernement allemand de faire la " transition énergétique ", on ne reconnait pas de changement d'opinion des opposants aux projets. Au mieux, ils se plaignent de « l'activisme » du gouvernement et de l'absence de plan pour la promotion du développement des énergies renouvelables, tout en soulignant qu'il existe encore de meilleures alternatives.

Même après la décision du gouvernement fédéral de sortir définitivement du nucléaire (" la transition énergétique») la discussion sur la politique énergétique reste très diversifiée. Il faut mentionner ici l'incertitude face à l'avenir des énergies alternatives, dans la mesure où des investissements dans une technologie peuvent après un court laps de temps, se révéler erronés et devenir des pertes de capitaux. Plutôt que de 
réaliser des centrales nationales de pompage-turbinage, il est ainsi recommandé de s'assurer la sécurité de réserves d'énergie dans les centrales correspondantes en Norvège (voir Asendorpf, 2011), ou par conversion en méthane dans le réseau de gaz naturel allemand (cf. Noir 2011) ; le projet Desertec déjà mentionné peut être une alternative au photovoltaïque national (voir Randow, 2011). Cependant, l'incertitude reste alimentée par des problèmes nouvellement découverts et évoqués dans les médias, en ce qui concerne les énergies renouvelables, tels que par exemple les modifications du microclimat (voir dpa 2012, p.20), ou la preuve scientifique de la perturbation du sommeil par les parcs éoliens (voir oV 2012, p.36).

Compte tenu des controverses du débat sur la politique énergétique, l'idée selon laquelle l'on sacrifie la qualité de vie pour une voie erronée semble s'imposer; ce qui prépare des conflits de type NIMBY. Rau, Walter et Zoellner (2011) soulignent l'importance des procédures et d'une certaine justice distributive. Les préoccupations concernant les paysages relèvent plutôt de la stratégie argumentaire (voir Leibenath et Otto 2012). Si dans la transition d'un système fordiste à un système post-fordiste de l'approvisionnement énergétique, les cartes sont redistribuées (en termes de " centralité», du nombre et de la taille des producteurs d'électricité, du rôle de l'État par rapport à l'influence des citoyens, des coûts et des revenus, etc.), la résistance est une option qui consiste à ne pas appartenir aux perdants (Weiss 2010, pp 30 et suivantes). Le partage des bénéfices dans des projets communautaires ne peut généralement pas faire disparaitre ces préoccupations. Pour de nombreux citoyens, les bénéfices fiscaux pour la "communauté» sont trop incertains et déjà trop impersonnels. Anticiper une adaptation du projet au contexte géographique et à la voie locale de développement ne peut être mené à bien que dans les grands projets.

\section{BIBLIOGRAPHY}

Asendorpf, D. (2011): Norwegen, der Akku Europas. Die Zeit 36, 01.09.2011, S. $37 f$.

Bosch, S. und G., Peyke (2011): Gegenwind für die Erneuerbaren - Räumliche Neuorientierung der Wind-, Solar- und Bioenergie vor dem Hintergrund einer verringerten Akzeptanz sowie zunehmender Flächennutzungskonflikte im ländlichen Raum. Raumforschung und Raumordnung (2), S. 105-118

Brücher, W. (2009): Energiegeographie. Wechselwirkungen zwischen Ressourcen, Raum und Politik. Berlin, Stuttgart

Deutscher Rat für Landespflege (2006): Die Auswirkungen erneuerbarer Energien auf Natur und Landschaft. Schriftenreihe des Deutschen Rats für Landespflege 79, S. 5-47

Deutsche Presse-Agentur dpa (2012): Große Windfarmen verändern das lokale Klima. Kölnische Rundschau 01.05.2012, S. 20

Hollersen, W. (2010): Gut gegen Gut. Der Spiegel (3), S. 40-43 
Jones, C.R. und J. R., Eiser (2010): Understanding ,local' opposition to wind development in the UK: How big is a backyard? Energy Policy (6), S. 3106-3117

Leibenath, M. und A., Otto (2012): Diskursive Konstituierung von Kulturlandschaft am Beispiel politischer Windenergiediskurse in Deutschland. Raumforschung und Raumordnung (2), S.

119-131

o.V. (2012): Schafdieb Windrad. Die Zeit Nr. 12, 15.03.2012, S. 36

Randow, G. v. (2011): Sonne statt Soldaten. Die Zeit 36, 01.09.2011, S. 32

Rau, Irina, G., Walter und J., Zoellner (2011): Wahrnehmung von Bürgerprotesten im Bereich erneuerbarer Energien: Von NIMBY-Opposition zu kommunaler Emanzipation.

Umweltpsychologie (2), S. 37-51

Schwarzer, C. (2011): Windkraft in die Kaverne. Die Zeit 37, 08.09.2011, S. 32

Trianel GmbH (Hrsg.) (2011): Projektentwicklung Wasserspeicherkraftwerke. Aachen

Weiss, G. (2010): Konflikte um Anlagen zur Energieerzeugung im Licht der Regulationstheorie. Schüssler, F. (Hrsg.): Geographische Energieforschung, Frankfurt a. M., S. 21-36

Weiss, G. (2008): Umweltkonflikte verstehen. Die Ansiedlung von Industriebetrieben im Spannungsfeld regionaler Entwicklungspfade und nationaler Umweltdiskussionen. München

\section{ABSTRACTS}

The expansion of renewable energies in Germany became partly restricted due to the protest of local population, living near designated power plant sites. The study of disputes concerning planned pump storage hydropower and open space photovoltaic power stations in Germany between 2009 and 2012 revealed two types of conflicts: Local conflicts, where the power plant is seen as a disturbance of local livelihoods and regional conflicts, where the power plant is seen as contradictory to a perceived regional path of (mainly economic) development. The national discussions on renewable energies, especially alternatives of energy policy and technologies are generally important for local as well as regional type conflicts.

L'expansion des énergies renouvelables en Allemagne a été en partie jugulée par les protestations des populations locales vivant près des sites choisis pour l'implantation des centrales de production. L'étude de conflits concernant des centrales hydroélectriques de pompage-turbinage et des sites d'implantation de parcs photovoltaïques entre 2009 et 2012 a révélé l'existence de deux types de conflits : les conflits locaux où la centrale de production est vue comme une perturbation pour les modes de vie locaux et les conflits régionaux où la centrale est considérée comme étant en contradiction avec la voie locale de développement économique. Les discussions nationales sur les énergies renouvelables, en particulier sur les technologies et les politiques énergétiques alternatives sont généralement importantes pour les conflits locaux comme pour les conflits régionaux.

Der Ausbau der erneuerbaren Energien in Deutschland wurde zum Teil eingeschränkt aufgrund der Proteste der lokalen Bevölkerung, die in der Nähe der Kraftwerksstandorte lebt. Das Studium der Rechtsstreitigkeiten über geplante Pumpspeicherkraft und Freiflächen PhotovoltaikKraftwerke in Deutschland zwischen 2009 und 2012 zeigte zwei Arten von Konflikten: Lokale Konflikte, wo das Kraftwerk als eine Störung der lokalen Lebensunterhalt wird und regionale Konflikte, wo das Kraftwerk zu sehen wird als im Widerspruch zu einer wahrgenommenen Regionalbahn (vor allem wirtschaftliche) Entwicklung gesehen. Die nationalen Diskussionen über 
erneuerbare Energien, vor allem Alternativen der Energiepolitik und Technologien sind für die lokale und regionale Konflikte Typ generell wichtig.

INDEX

Mots-clés: centrale de pompage-turbinage, conflit d'usage de l'espace, parc photovoltaïque Keywords: conflict of use of space, open space photovoltaic power station, pump storage hydropower

Schlüsselwörter: Flächennutzungskonflikt, Photovoltaïkanlage, Pumpspeicherkraftwerk

\section{AUTHOR}

GÜNTER WEISS

Universität Köln - g.weiss@uni-koeln.de 